\title{
Crystallization of recombinant Bacteroides fragilis glutamine synthetase (GlnN) isolated using a novel and rapid purification protocol
}

\author{
J.M. van Rooyen ${ }^{\text {b,* }}$, V.R. Abratt ${ }^{\mathrm{b}}$, H. Belrhali ${ }^{\mathrm{c}}$, B.T. Sewell $^{\mathrm{a}}$ \\ ${ }^{a}$ Electron Microscope Unit, University of Cape Town, South Africa \\ ${ }^{\mathrm{b}}$ Department of Molecular and Cell Biology, University of Cape Town, South Africa \\ ${ }^{\mathrm{c}}$ European Molecular Biology Laboratory, Grenoble, France
}

\section{A R T I C L E I N F O}

\section{Article history:}

Received 28 April 2010

and in revised form 4 June 2010

Available online 22 June 2010

\section{Keywords:}

Glutamine synthetase type III

GSIII

Structure

Bacteroides fragilis

X-ray crystallography

Protein purification

\begin{abstract}
A B S T R A C T
Glutamine synthetase enzymes (GSs) are large oligomeric enzymes that play a critical role in nitrogen metabolism in all forms of life. To date, no crystal structures exist for the family of large ( $\sim 1 \mathrm{MDa})$ type III GS enzymes, which only share $9 \%$ sequence identity with the well characterized GSI and GSII enzymes. Here we present a novel protocol for the isolation of untagged Bacteroides fragilis GlnN expressed in an auxotrophic Escherichia coli strain. The rapid and scalable two-step protocol utilized differential precipitation by divalent cations followed by affinity chromatography to produce suitable quantities of homogenous material for structural characterization. Subsequent optimizations to the sample stability and solubility led to the discovery of conditions for the production of the first diffraction quality crystals of a type III GS enzyme.
\end{abstract}

(c) 2010 Elsevier Inc. All rights reserved.

\section{Introduction}

Glutamine synthetases (GSs) ${ }^{1}$ are large oligomeric enzymes that play a central role in nitrogen assimilation, catalysing the condensation of ammonium and glutamate to form glutamine, a precursor for the synthesis of many critical bio-molecules. The ancient [1] and ubiquitous [2] GS superfamily is, therefore, evolutionarily diverse and can be divided into three main groupings, namely GSI, GSII and GSIII, on the basis of amino-acid divergence [3].

GSI and GSII enzymes have been purified from numerous bacterial and eukaryotic sources, respectively, over the past five decades of biochemical and structural investigations into their functioning and regulation [4]. The first crystal structure of a GSI enzyme [5] was solved using material prepared from an adenylylation deficient Salmonella typhimurium strain using a combination of simple differential precipitation techniques and nucleotide-analogue affinity chromatography [6]. Since then, advances in recombinant DNA technology and the advent of affinity purification technologies have greatly facilitated the isolation of GS material suitable for crystallization. The first crystal structure of a GSII enzyme

\footnotetext{
* Corresponding author. Address: Electron Microscope Unit, University of Cape Town, Private Bag X3, Rondebosch 7701, South Africa. Fax: +27 0216891528.

E-mail address: jason@science.uct.ac.za (J.M. van Rooyen).

1 Abbreviations used: GSs, glutamine synthetases; CFE, cell-free extract; AGB, AffiGel Blue; MetSox, methionine sulphoximine; GGT, $\gamma$-glutamyl transferase; ANX, anion-exchange chromatography; SEC, size-exclusion chromatography; HIC, hydrophobic interaction chromatography; EM, electron microscopy.
}

was of the Zea mays GS1a protein [7], which was heterologously expressed in Escherichia coli and purified using a combination of anion exchange and affinity chromatography steps [8]. More recently, several structures of GSII enzymes purified with affinity tags $[9,10]$ have been reported.

In comparison, GSIII enzymes have only been isolated and characterized from a small number of sources [11-13] despite the widespread occurrence of homologues in a number of evolutionarily divergent organisms [14-16], including, the anaerobic pathogenic protozoan Trichomonas vaginalis [17]. To date, the only structural information describing the large GSIII enzymes ( $\sim 1 \mathrm{MDa}$ ), which share only $\sim 9 \%$ sequence identity with the GSI enzymes, is from a low-resolution cryo-EM reconstruction of the GlnN enzyme from the opportunistic human pathogen Bacteroides fragilis [3]. A detailed understanding of the functioning and evolution of these divergent enzymes in light of the known structurefunction relationships of the other GS families is, therefore, still lacking. Although the current purification protocols [3,11] are sufficient for the preparation of small quantities of material for microscopy and biochemical studies, the poor yields, resulting from protein solubility and stability issues, represent a barrier to the attainment of suitable material for crystallization and the achievement of these goals.

Here we present a novel scheme for the rapid isolation of suitable quantities of pure GlnN protein and the subsequent sample optimizations that have lead to the first diffraction quality crystals of a GSIII enzyme. 


\section{Materials and methods}

All reagents and chemicals were sourced from Sigma-Aldrich unless otherwise stated.

\section{Expression and cell lysis}

The glutamine synthetase from $B$. fragilis BF-1, GlnN, was expressed constitutively from a pEcoR1-derived low copy number plasmid, pJS139, in an auxotrophic E. coli strain, YMC11 (gln $\mathrm{A}^{-}$, $\mathrm{ntrB}^{-}$, ntrC ${ }^{-}, \mathrm{ApS}$ ) [18] as described previously [3] but without the low-nitrogen induction and CTAB steps. Following $16 \mathrm{~h}$ growth in Luria broth [19] containing ampicillin $(100 \mu \mathrm{g} / \mathrm{ml})$ at $37^{\circ} \mathrm{C}$ with aeration, cells were collected by centrifugation for $15 \mathrm{~min}$ at $7500 \mathrm{rpm}$ at $4{ }^{\circ} \mathrm{C}$ and resuspended in $1 / 50$ th of the original culture volume of extraction buffer ( $50 \mathrm{mM}$ imidazole, $50 \mathrm{mM} \mathrm{KCl}, \mathrm{pH} 7.1$ ). Cells were then disrupted by sonication for $4 \mathrm{~min}$ on ice using a Misonix sonicator fitted with a microtip operating at a power output of $9 \mathrm{~W}$ and cell-free extract (CFE) was then prepared by centrifugation at $15,000 \mathrm{rpm}$ for $30 \mathrm{~min}$ at $4{ }^{\circ} \mathrm{C}$. For long-term storage, CFE was diluted 1:2 with glycerol, aliquoted into Sterilin ${ }^{\circledR}$ plastic tubes, flash-frozen in liquid nitrogen, and then placed at $-80^{\circ} \mathrm{C}$.

\section{Protein purification}

Frozen CFE was thawed and diluted 1:2 with extraction buffer ( $50 \mathrm{mM}$ imidazole, $50 \mathrm{mM} \mathrm{KCl}, \mathrm{pH}$ 7.1). $\mathrm{ZnCl}_{2}$ was then added to a final concentration of $2 \mathrm{mM}$ and the preparation was left to precipitate for $1 \mathrm{~h}$ at room temperature. Precipitated material was collected by centrifugation at $15,000 \mathrm{rpm}$ at $4{ }^{\circ} \mathrm{C}$ for $30 \mathrm{~min}$ and resuspended, using a Dounce homogenizer, in ice-cold wash buffer (15 mM imidazole, $2 \mathrm{mM} \mathrm{MgCl}_{2}, \mathrm{pH} 7.1$ ). Following recollection of the washed material, a solubilization step was performed in resuspension buffer ( $50 \mathrm{mM}$ imidazole, $500 \mathrm{mM} \mathrm{KCl}, 10 \%$ glycerol, $\mathrm{pH}$ 7.1). Insoluble material remaining after $1 \mathrm{~h}$ at $4{ }^{\circ} \mathrm{C}$ was removed by a 30 -min centrifugation step at $14,000 \mathrm{rpm}$ in a desktop centrifuge at $4{ }^{\circ} \mathrm{C}$. Before loading onto the pre-equilibrated $5 \mathrm{ml}$ Affi-Gel Blue (AGB) HiTrap affinity column (GE Healthcare), the supernatant was diluted 1:10 with ice-cold AGB equilibration buffer (50 mM imidazole, $\mathrm{pH} 7.1,50 \mathrm{mM} \mathrm{KCl}, 20 \mathrm{mM} \mathrm{MgCl}_{2}, 10 \%$ glycerol, and $0.02 \%$ sodium azide) and filtered through a $0.45-\mu \mathrm{m}$ filter. The column was then washed with 5 volumes of equilibration buffer before elution of the bound protein with 2 volumes of equilibration buffer containing $10 \mathrm{mM}$ ATP. A period of $5 \mathrm{~min}$ was allowed to elapse between the application of the first and second volumes of elution buffer. All chromatography steps were carried out at a flow rate of $1 \mathrm{ml} / \mathrm{min}$ at ambient temperature.

After chromatographic purification, methionine sulphoximine (MetSox) was added to the eluted fraction at a final concentration of $8 \mathrm{mM}$ and the sample was stored for $16 \mathrm{~h}$ at $4{ }^{\circ} \mathrm{C}$. This final preparation was concentrated to $6 \mathrm{mg} / \mathrm{ml}$ using Microsep and Nanosep 10 kDa MWCO centrifugal concentrators (Pall Corporation). The final preparation was divided into $10 \mu \mathrm{l}$ aliquots and flash-frozen in NUNCTM CryoTubes $^{\mathrm{TM}}$ (Thermo Fisher) for storage under liquid nitrogen.

\section{Biochemical assays and protein identification}

Protein concentrations were estimated using the Bradford assay [20] with Bio-Rad reagents and bovine serum albumin standards. Results of assays prepared in 96 well NUNCTM plates were read using a Titertek Multiscan plus MKII plate reader fitted with a 595-nm filter and data processing was carried out with Genesis Lite.
GS activity was assayed by the $\gamma$-glutamyl transferase (GGT) assay as described by Bender et al. [21] and subsequently modified by Southern et al. [11]. Total assay volumes including reaction mixtures and samples were also reduced from $1.5 \mathrm{ml}$ to $250 \mu \mathrm{l}$ to allow measurement in the Titertek Multiscan plate reader. Specific activity was expressed as $\mu$ moles of glutamyl hydroxamate formed per min, per mg protein, and $1 \mu \mathrm{mol}$ of glutamyl hydroxamate gave 2.916 absorbance units at a wavelength of $540 \mathrm{~nm}$.

Discontinuous SDS-PAGE was carried out according to Laemmli [22] using a Mini-PROTEAN 3 cell from Bio-Rad. Acrylamide (19:1 monomer:cross-linker) was used to prepare $5 \%$ stacking gels and 7.5 or $10 \%$ separating gels. Pre-stained molecular weight markers (Fermentas Life Sciences) were included in all electrophoretic separations. Gels were stained either with $0.25 \%$ Coomassie Brilliant Blue R250 in 45\% methanol and 10\% acetic acid or by a non-ammoniacal silver-staining protocol [23] using reagents from the GE Healthcare PlusOne ${ }^{\mathrm{TM}}$ Silver-Staining Kit. Protein purity was estimated by densitometry using the program IMAGEJ [24]. Peak areas, corresponding to bands in the lane under investigation, were calculated after baseline subtraction, and the purity of the GlnN band was determined from the ratio of its peak area to the total for all the bands in the lane.

\section{Crystallization and preliminary diffraction characterization}

$\mathrm{G} \ln N$, stored under liquid nitrogen, was thawed, diluted to $2 \mathrm{mg} / \mathrm{ml}$ with AGB equilibration buffer, and clarified by centrifugation at $14,000 \mathrm{rpm}$ in desktop centrifuge prior to the setting up of modified microbatch crystallization conditions [25]. Equal volumes $(1 \mu \mathrm{l}$ each) of protein and precipitant (reagent 20 from Hampton Research Crystal Screen diluted 1:2 with distilled $\mathrm{H}_{2} \mathrm{O}$ ) were dispensed under $5 \mathrm{ml} \mathrm{Al's} \mathrm{oil} \mathrm{(Hampton} \mathrm{Research)} \mathrm{in}$ 72 well microbatch plates (Greiner) and stored at ambient temperature $\left(21-23^{\circ} \mathrm{C}\right)$. All assessments of crystal growth and manipulations were performed on a Leica MZ6 stereo microscope fitted with a CLS 150X cold light source. Photographs of crystals were captured with a Zeiss Axiocam attached to a Nikon Diaphot inverted microscope fitted with a $20 \times$ short working distance objective lens.

The identity of the proteins comprising the crystals was confirmed using silver-stained SDS-PAGE. Crystals were washed serially in four droplets $(2 \mu \mathrm{l})$ of crystallization solution (Hampton condition 20 diluted $1: 2$ with $\mathrm{H}_{2} \mathrm{O}$ ) placed under oil in the microbatch plate and finally dissolved in a drop of diluted SDS-PAGE loading buffer $\left(1: 2 \mathrm{H}_{2} \mathrm{O}\right)$ before electrophoretic analysis.

Diffraction analyses were carried out on BM14 at the ESRF (Grenoble). Crystals were looped using LithoLoops ${ }^{\mathrm{TM}}$ fitted in Hampton HT Crystal Caps and cryo-protected with Paratone-N oil (Hampton Research) prior to being flash-cooled in liquid nitrogen. Data $\left(1^{\circ}\right.$ oscillations) were collected at $100 \mathrm{~K}$ using $0.95373 \AA$ wavelength X-rays (200 mA beam current) with an exposure of $120 \mathrm{~s}$ and a crystal-to-detector distance of $324.8 \mathrm{~mm}$.

\section{Electron microscopy of GlnN crystals}

Several microlitres of crystallization solution were removed and applied to EM grids coated with a glow-discharged thin carbon support film, before being stained with $2 \%$ uranyl acetate solution using the droplet method [26]. Electron micrographs were recorded by a Proscan $2048 \times 2048$ slow-scan CCD camera, with $14 \mu \mathrm{m}$ pixels, using a Leo 912 TEM operating at $120 \mathrm{kV}$ with zero-loss energy filtering. Fourier transforms were calculated using a FFT plug-in [27] in Paint Shop Pro ${ }^{\circledR}$ and patch correlation averages were calculated in 2DX after Fourier filtering [28]. 


\section{Results and discussion}

Two major limitations characterized the previously reported isolations of GlnN from the heterologous YMC11 E. coli (pJS139) expression system, namely, limited solubility and the co-purification of large molecular weight contaminating complexes. Several alternate purification technologies and strategies were, therefore, evaluated for their ability to address these issues. These techniques included differential PEG precipitations, ANX Sepharose anion-exchange chromatography (ANX), HR Sephacryl size-exclusion chromatography (SEC), nucleotide-analogue dye affinity chromatography (AF) matrices including Affi-Gel Blue (AGB) and Reactive Red Sepharose, and Butyl Sepharose hydrophobic interaction chromatography (HIC) (data not shown). The final purification scheme presented below is a result of these optimizations, which paralleled the screening of crystallization conditions. Over the course of the experiment, information flowed in both directions in an iterative fashion to inform decisions about the protein purification strategy and crystallization conditions.

\section{Solubility enhancements}

During the purification optimizations, GlnN, displayed a propensity to precipitate out of solution as previously noted by van Rooyen et al. [3]. Fibrous aggregates of GlnN, identified by SDSPAGE, routinely appeared upon concentration by ultra-filtration and in even more dilute fractions such as the elutions from ANX $\mathrm{AF}$, and SEC. In addition to these "spontaneous" precipitation events, information about the limited solubility of GlnN under more specific conditions emerged from the PEG fractionation steps and the numerous crystallization trials (see below). These results suggested that GlnN was very sensitive to precipitation by moder- ate molecular weight polymers such as PEG and at $\mathrm{pH}$ values below 5 . At the same time, a systematic investigation of the solubility limiting effects of various ionic species in the extraction solutions revealed that, as in the case of GSI [29], GlnN, was particularly sensitive to precipitation by divalent cations. This behaviour could be prevented in the presence of moderate salt concentrations, such as found in the crystallization conditions $(0.2 \mathrm{M}$ ammonium sulphate), and $\mathrm{pH}$ values above 4.6 but below 5.8 . This partially reversibility was, therefore, exploited to rapidly purify the $G \ln N$ enzyme as inspired by the previously reported scheme for isolating GSI using $\mathrm{Zn}^{2+}$ precipitation [29]. The method presented here, however, differs in several regards: (1) only the $\mathrm{Zn}^{2+}$ fractionation was used; (2) precipitation took place at $\mathrm{pH} 7$ and not under acidic conditions; (3) the wash solution contained only $2 \mathrm{mM} \mathrm{Mg}^{2+}$ instead of $50 \mathrm{mM}$; and (4) the resuspension volumes and the salt concentrations were optimized for the recovery of $\mathrm{GlnN}$ from the $\mathrm{Zn}^{2+}$ pellet. During the latter optimizations it was also found that high salt concentrations condensed the remaining insoluble fraction, thus, facilitating the recovery of soluble GlnN.

Although the sensitivity to precipitation by divalent cations proved useful for the isolation of pure GlnN (see below), the phenomenon did not explain the spontaneous losses seen in dilute solutions of the semi-pure protein or the losses observed in the absence of such ions (data not shown). Solubility enhancing additives were, therefore, sought in order ameliorate the losses encountered during the purifications and to overcome the presumed barrier to crystallization caused by the limited solubility of GlnN. Of these, glycerol was found to have the greatest effect on GlnN solubility. The first indication of this came from crystallization trials setup in the presence of $10 \%$ glycerol in an attempt to reduce nucleation of micro-crystals (see below). At this concentration, glycerol prevented all protein precipitation, even at high PEG concentrations,
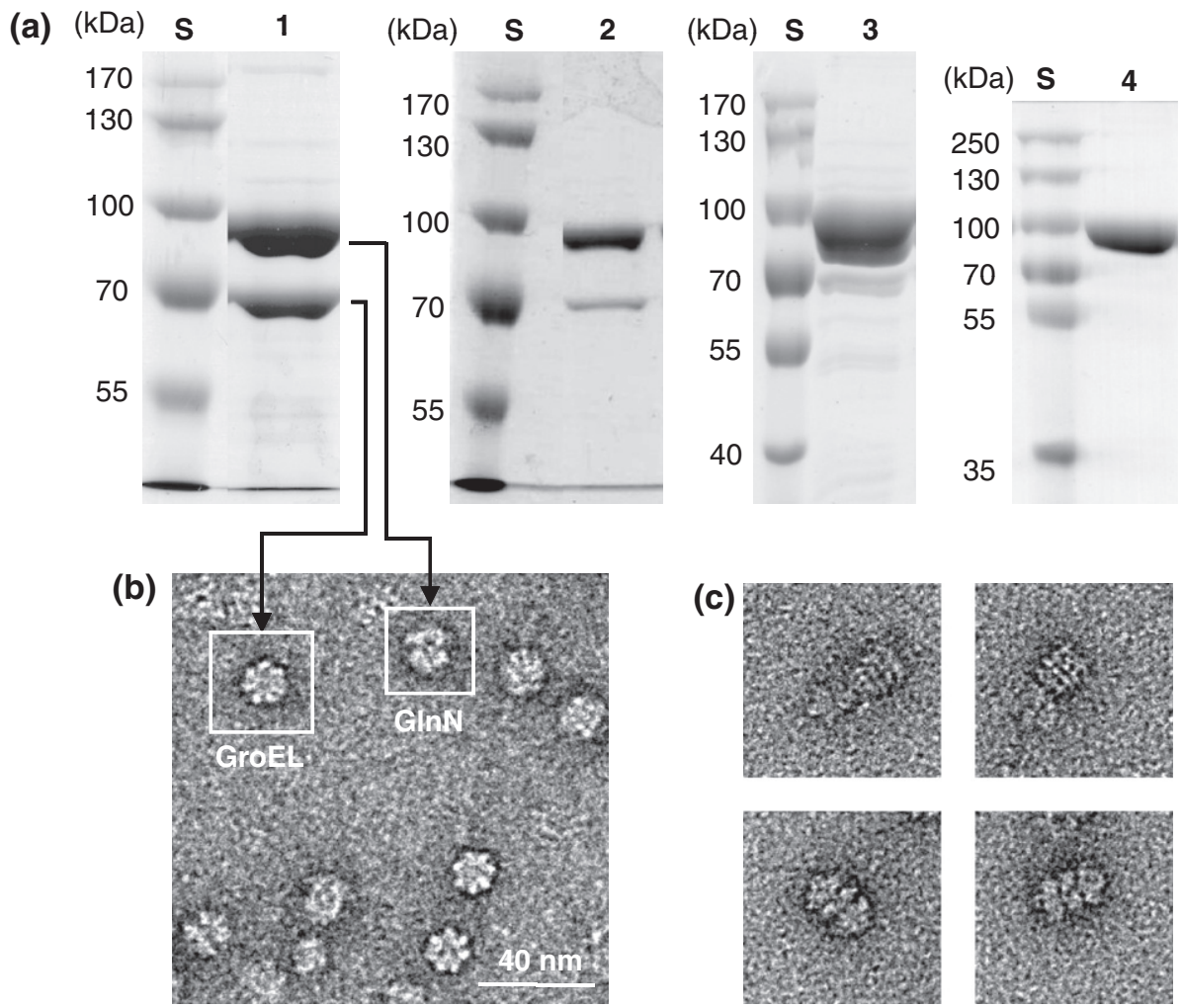

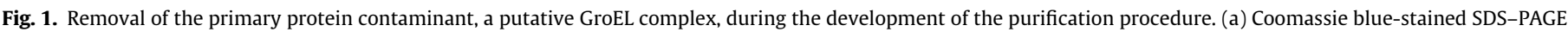

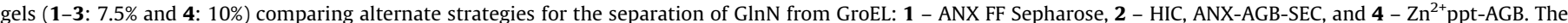

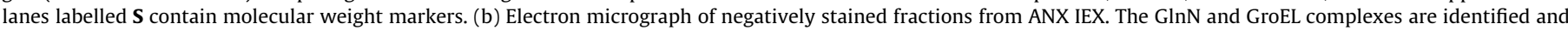

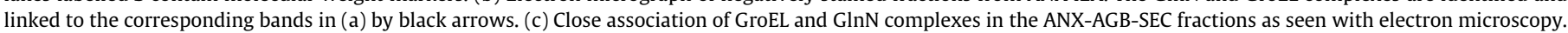


and the additive was, therefore, included in all future purification and crystallization solutions. The cryo-protective abilities of glycerol also permitted the long-term storage of the final GlnN crystallization stocks in liquid nitrogen.

\section{Removal of large protein contaminants}

The second major problem thought to be hindering the crystallization of $\mathrm{GlnN}$ was the inability of the early purification procedures to separate $\mathrm{GlnN}$ from a similarly sized contaminating protein complex. This complex, which co-eluted with GlnN from a number of chromatographic matrices (Fig. 1a), was putatively identified as GroEL on the basis of its SDS-derived molecular weight and characteristic geometry, as seen with electron microscopy (EM) (Fig. 1b). The EM analyses also revealed a close association between the GlnN and GroEL complexes purified by the sequential ANX-AGB-SEC method (Fig. 1c). GS-GroEL associations have been noted before $[30,31]$ and could be the reason for their co-elution from the various chromatographic matrices. The presence of this contaminant, even at trace levels, was particular concerning because the reported crystallization conditions for the native protein from E. coli [32] were very similar to the conditions that produced the majority of crystallization phenomena (see below). The two most successful methods for removing GroEL were the already proven AGB AF [30] and HIC [32] separations (Fig. 1a). The latter scheme together with a SEC purification step proved quite successful, however, the large losses observed during the three concentration steps precluded its usefulness for preparing the large quantities of protein needed for crystallization trials. The AGB affinity step was, therefore, combined with the $\mathrm{Zn}^{2+}$ precipitation method to produce the final purification protocol (see below), which showed almost no GroEL contamination (Fig. 1a).

\section{Final purification protocol}

Several additional enhancements were made to the purity and stability of the GlnN preparation protocols. Firstly, the purity of the fraction eluted from the Affi-Gel Blue affinity matrix was improved by changing from an ionic based elution to a more specific competitive elution with ATP. This alteration also facilitated the next improvement which was the inhibition of GlnN by the well known GS inhibitor MetSox, the aim of which was the improved stability of the final preparation. The purity of the affinity fraction was also sufficiently high that the additional selectivity of the 100 kDa MWCO filter was unnecessary. A 10-kDa MWCO filter was used instead, thus, allowing increased centrifugal speeds and, therefore, faster isolations of higher concentrations of protein $(6-8 \mathrm{mg} / \mathrm{ml})$ for storage and crystallization.

A summary of the final purification scheme is given in Table 1 and representative samples taken during the purification process are shown in Fig. 2. It can be seen from these results that pure GlnN was isolated quickly and easily with this method. The $\mathrm{Zn}^{2+}$ precip- itation step alone was able to produce $63 \%$ pure GlnN at concentrations as high as $6 \mathrm{mg} / \mathrm{ml}$ (as measured by densitometry of all protein bands above $25 \mathrm{kDa}$ ). The subsequent affinity purification step removed all traces of contaminants and resulted in a 22 -fold total enrichment of the GlnN above level found in the starting material. The disagreement between the SDS-PAGE and specific activity derived purity values, 10 and 1.4 -fold, respectively, suggested that the affinity purification step also removes a large fraction of inactive but soluble GlnN material. Together with the interactions with GroEL noted above, these results suggest that the spontaneous aggregation of $\mathrm{GlnN}$ was caused by the association of denatured molecules.

Unfortunately, this high purity came at the cost of a poor yield, with losses occurring primarily at the $\mathrm{Zn}^{2+}$ precipitation (77\% loss), AGB AF (additional 9\% loss), and final concentration steps (additional $8.5 \%$ loss). Nonetheless, these losses were tolerable considering the rapidity, ease, and scalability of the purification method. As demonstrated here, despite only recovering $5.5 \%$ of the total enzyme units from a modest $44 \mathrm{ml}$ of CFE, the protocol produced enough pure $\mathrm{GlnN}$ enzyme for $\sim 300$ batch crystallizations (each requiring $1 \mu \mathrm{l} @ 2 \mathrm{mg} / \mathrm{ml}$ ).

\section{Crystallization of full-length GlnN}

The optimizations to the purification protocol produced several different preparations with varying purities, stabilities, and solute compositions. From the outset, attempts were made to crystallize these preparations and the successes of the optimizations were

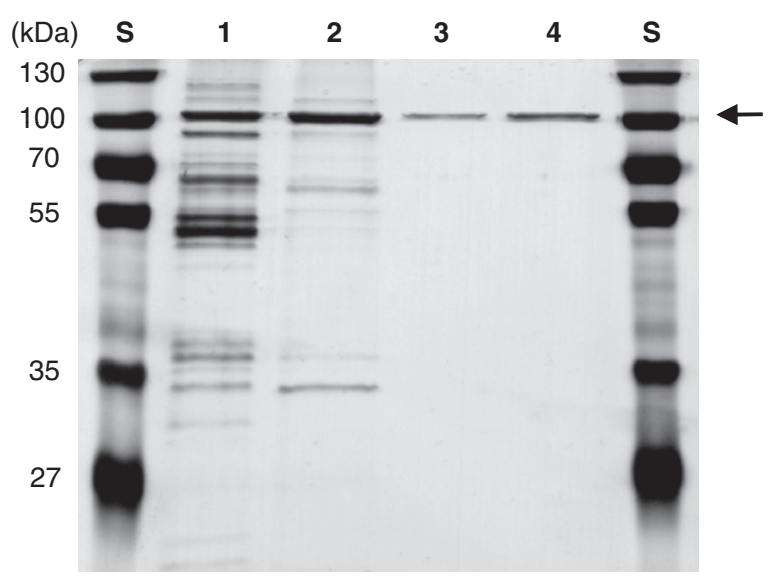

Fig. 2. Summary of the novel two-step purification of GlnN from E. coli YMC11 Samples from each purification step are shown on a silver-stained SDS-PAGE gel (10\%): 1 - CFE $(0.67 \mu \mathrm{g}), \mathbf{2}$ - resolubilized $\mathrm{ZnCl}_{2}$ precipitate $(0.33 \mu \mathrm{g}), \mathbf{3}$ - AGB eluent $(0.07 \mu \mathrm{g})$, and $\mathbf{4}$ - ultrafiltration retentate $(0.07 \mu \mathrm{g})$. Lanes labelled $\mathbf{S}$ contained molecular weights markers and the position of the GlnN monomer is shown by an arrow. The colour image of the silver-stained gel was split into RGB channels and the grey levels of the blue channel were adjusted for presentation purposes but no bands in the gel image were masked by this alteration.

Table 1

Purification statistics for production of recombinant GlnN from E. coli YMC11.

\begin{tabular}{|c|c|c|c|c|c|c|c|}
\hline Purification step & Volume (ml) & Total protein (mg) & Total enzyme activity $(U)^{a}$ & Specific activity (U/mg) & Yield (\%) & Purity $^{\mathrm{b}}(\%)$ & Purification fold ${ }^{\mathrm{c}}$ \\
\hline Clarified cell lysate & 44 & 241 & 129 & 0.534 & 100 & 6 & 1 \\
\hline Solubilized $\mathrm{Zn}^{2+}$ precipitate & 50 & 38.7 & 29.2 & 0.754 & 23 & 63 & 1.4 \\
\hline AGB affinity eluent & 9 & 1.59 & 17.5 & 11.0 & 14 & 100 & 20.6 \\
\hline Ultra-filtration retentate $e^{d}$ & 0.105 & 0.65 & $\mathrm{n} / \mathrm{a}$ & & & & \\
\hline
\end{tabular}

Values in the table represent the average of at least two measurements and all assays were carried out at dilutions within their linear response regions.

a Activity is expressed as $\mu$ moles of glutamyl hydroxamate formed per min.

b Purity was calculated from densitometry of bands in Coomassie-stained 10\% SDS-PAGE gels.

c Purification fold $=$ specific activity/specific activity of clarified cell lysate.

d The pooled AGB ATP eluent was treated with MetSox before incubation for $16 \mathrm{~h}$ at $4{ }^{\circ} \mathrm{C}$. 
(a)

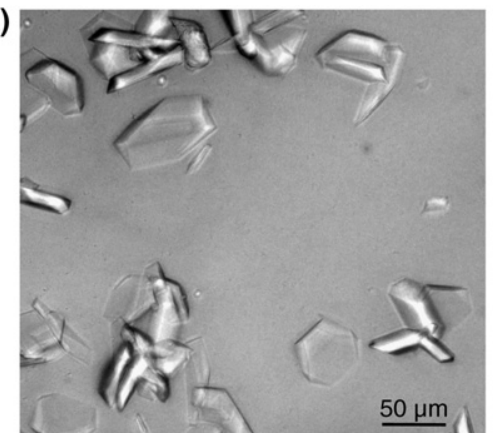

(b)

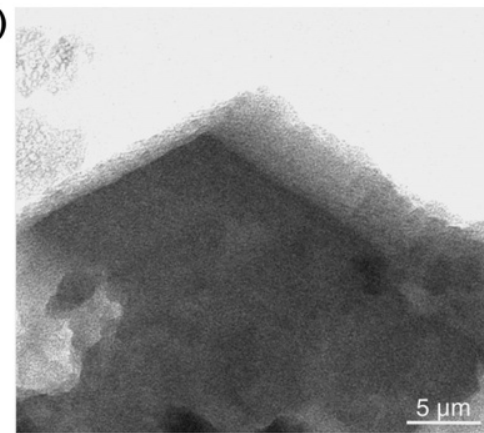

(c)

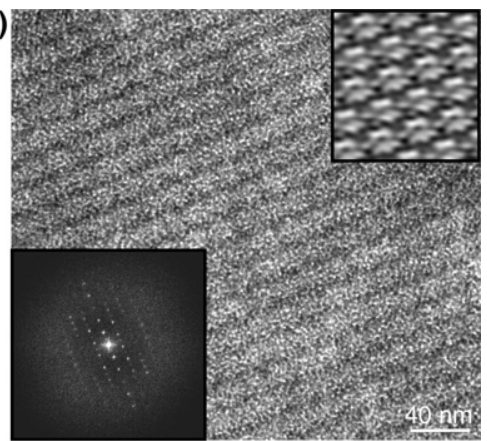

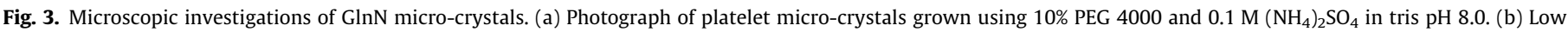

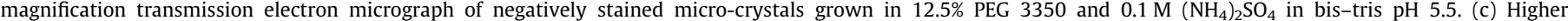

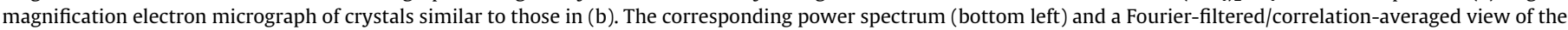
crystal lattice (upper right) are shown as insets. Grey levels were adjusted for presentation purposes.

(a)

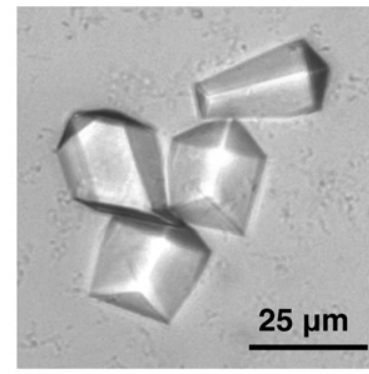

(b)

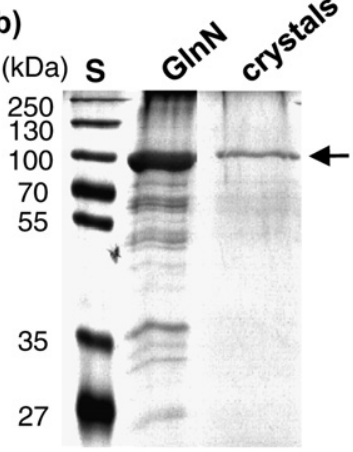

(c)

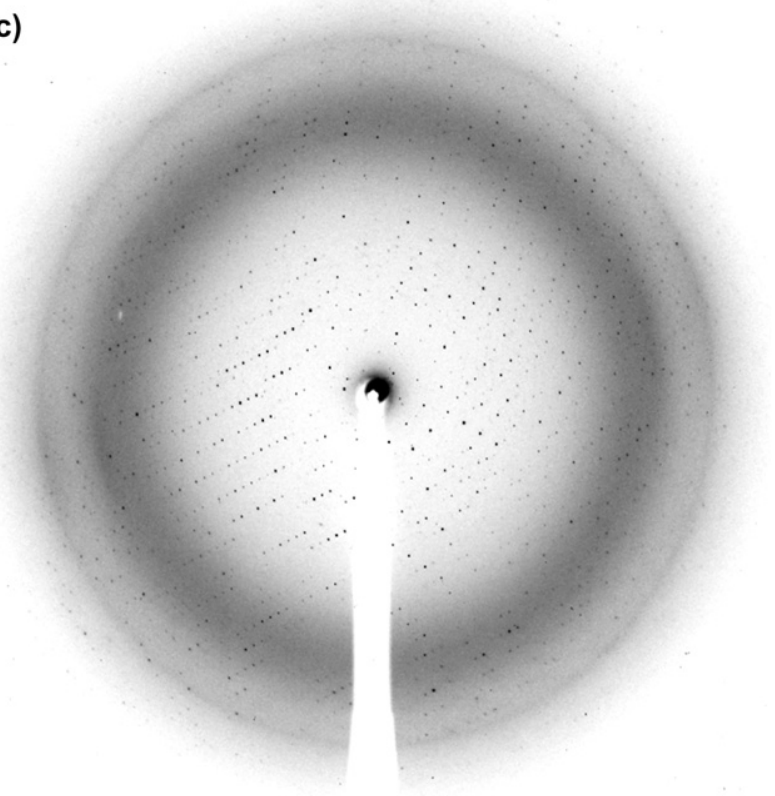

Fig. 4. Growth, identification, and characterization of GlnN crystals. (a) Photograph of GlnN crystals grown over several days following streak seeding. (b) Silver-stained SDS-PAGE gel (10\%) identifying the crystals (five washed $0.8 \mu \mathrm{m}$ crystals) as GInN $(1.9 \mu \mathrm{g}$ of crystallization stock). Molecular weight markers were loaded into the lane marked S. (c) Diffraction (3 Å resolution) from a cryo-protected P1 GlnN crystal. The grey levels in images (a) and (c) were adjusted for presentation purposes. The colour image of the silver-stained gel in (b) was split into RGB channels and the blue channel was adjusted for contrast but no bands in the gel image were masked by this alteration.

partly evaluated based on the feedback from the crystallization trials. The commercial screens yielded a number of promising leads and for the most part, these were achieved with the PEG 3350/ 4000 precipitants and manifested as showers of plate-like microcrystals (Fig. 3). The growth of crystals following precipitation, their small size, and the numerous macro-seedings necessary to enlarge them all suggested that only a sub-fraction of GlnN molecules were forming the crystals and this was, therefore, a major barrier to the attainment of large, well-diffracting crystals.

Following the improvements to the purity, solubility and stability of the GlnN protein preparations (described above), complete re-screening using a commercial matrix (Hampton Crystal Screen) yielded much improved crystallization conditions. As before, the initial screening results produced a large number of precipitation phenomena, particularly in the PEG conditions, but subsequent optimizations of the protein and precipitant concentrations produced irregular protein crystals after 4 days of growth for a diluted Hampton condition 20: $0.1 \mathrm{M}$ ammonium sulphate, $50 \mathrm{mM}$ sodium acetate trihydrate $\mathrm{pH} 4.6$, and $12.5 \%$ PEG 4000 . After mapping the meta-stable zone, streak-seeding produced single regular monoclinic crystals (Fig. 4a) and these were conclusively identified as GlnN by SDS-PAGE (Fig. 4b). Crystals diffracting to $3.0 \AA$ in a space group of $P 1$ (with unit cell dimensions $a=141.79 \AA, b=148.30 \AA$, $\left.c=59.32 \AA, \alpha=115.76^{\circ}, \beta=93.65^{\circ}, \gamma=115.99^{\circ}\right)$ were routinely produced following several days of growth (Fig. 4c).

\section{Conclusions}

The work presented here has addressed the shortcomings of the currently available methods for the isolation of suitable GlnN samples for structural characterization. It has been demonstrated that by exploiting the partially reversible precipitation of GlnN by $\mathrm{Zn}^{2+}$ ions and the selectivity of nucleotide-analogue affinity chromatography, it is possible to produce sufficient quantities of highly pure material for crystallization. The resultant improvements to the purity, solubility, and stability of the GlnN material achieved with the new method have also lead to the discovery of the conditions for the production of diffraction quality crystals of ATP- and MetSox-bound GlnN. The preliminary data collected from these crystals is promising ( $3 \AA$ resolution) and will, in future work, be used to solve the first crystal structure of a type III GS enzymes.

\section{Acknowledgments}

This work was supported by the National Research Foundation of South Africa. Synchrotron access was supplied by the European Molecular Biology Laboratory and Mr. Joachim Fleury helped with the data collection. J.v.R acknowledges Ph.D. scholarships from the NRF and the University of Cape Town. 


\section{References}

[1] Y. Kumada, D.R. Benson, D. Hillemann, T.J. Hosted, D.A. Rochefort, C.J. Thompson, W. Wohlleben, Y. Tateno, Evolution of the glutamine synthetase gene, one of the oldest existing and functioning genes, Proc. Natl. Acad. Sci. USA 90 (1993) 3009-3013.

[2] G. Pesole, M.P. Bozzetti, C. Lanave, G. Preparata, C. Saccone, Glutamine synthetase gene evolution: a good molecular clock, Proc. Natl. Acad. Sci. USA 88 (1991) 522-526.

[3] J.M. van Rooyen, V.R. Abratt, B.T. Sewell, Three-dimensional structure of a type III glutamine synthetase by single-particle reconstruction, J. Mol. Biol. 361 (2006) 796-810.

[4] D. Eisenberg, H.S. Gill, G.M. Pfluegl, S.H. Rotstein, Structure-function relationships of glutamine synthetases, Biochim. Biophys. Acta 1477 (2000) $122-145$.

[5] R.J. Almassy, C.A. Janson, R. Hamlin, N.H. Xuong, D. Eisenberg, Novel subunitsubunit interactions in the structure of glutamine synthetase, Nature 323 (1986) 304-309.

[6] C.A. Janson, P.S. Kayne, R.J. Almassy, M. Grunstein, D. Eisenberg, Sequence of glutamine synthetase from Salmonella typhimurium and implications for the protein structure, Gene 46 (1986) 297-300.

[7] H. Unno, T. Uchida, H. Sugawara, G. Kurisu, T. Sugiyama, T. Yamaya, H. Sakakibara, T. Hase, M. Kusunoki, Atomic structure of plant glutamine synthetase: a key enzyme for plant productivity, J. Biol. Chem. 281 (2006) 29287-29296.

[8] H. Sakakibara, H. Shimizu, T. Hase, Y. Yamazaki, T. Takao, Y. Shimonishi, T. Sugiyama, Molecular identification and characterization of cytosolic isoforms of glutamine synthetase in maize roots, J. Biol. Chem. 271 (1996) 29561-29568.

[9] W.W. Krajewski, R. Collins, L. Holmberg-Schiavone, T.A. Jones, T. Karlberg, S.L. Mowbray, Crystal structures of mammalian glutamine synthetases illustrate substrate-induced conformational changes and provide opportunities for drug and herbicide design, J. Mol. Biol. 375 (2008) 217-228.

[10] Y.X. He, L. Gui, Y.Z. Liu, Y. Du, Y. Zhou, P. Li, C.Z. Zhou, Crystal structure of Saccharomyces cerevisiae glutamine synthetase Gln1 suggests a nanotube-like supramolecular assembly, Proteins 76 (2009) 249-254.

[11] J.A. Southern, J.R. Parker, D.R. Woods, Expression and purification of glutamine synthetase cloned from Bacteroides fragilis, J. Gen. Microbiol. 132 (1986) 28272835.

[12] M. Garcia-Dominguez, J.C. Reyes, F.J. Florencio, Purification and characterization of a new type of glutamine synthetase from cyanobacteria, Eur. J. Biochem. 244 (1997) 258-264.

[13] K.R. Amaya, S.A. Kocherginskaya, R.I. Mackie, I.K.O. Cann, Biochemical and mutational analysis of glutamine synthetase type III from the rumen anaerobe Ruminococcus albus 8, J. Bacteriol. 187 (2005) 7481-7491.

[14] J. Xu, M.K. Bjursell, J. Himrod, S. Deng, L.K. Carmichael, H.C. Chiang, L.V. Hooper, J.I. Gordon, A genomic view of the human - Bacteroides thetaiotaomicron symbiosis, Science 299 (2003) 2074-2076.

[15] O. White, J.A. Eisen, J.F. Heidelberg, E.K. Hickey, J.D. Peterson, R.J. Dodson, D.H. Haft, M.L. Gwinn, W.C. Nelson, D.L. Richardson, K.S. Moffat, H. Qin, L. Jiang, W.
Pamphile, M. Crosby, M. Shen, J.J. Vamathevan, P. Lam, L. McDonald, T. Utterback, C. Zalewski, K.S. Makarova, L. Aravind, M.J. Daly, K.W. Minton, R.D. Fleischmann, K.A. Ketchum, K.E. Nelson, S. Salzberg, H.O. Smith, J.C. Venter, C.M. Fraser, Genome sequence of the radioresistant bacterium Deinococcus radiodurans $\mathrm{R} 1$, Science 286 (1999) 1571-1577.

[16] D.L. Robertson, R.S. Alberte, Isolation and characterization of the glutamine synthetase from the marine diatom Skeletonema costatum, Plant Physiol. 111 (1996) 1169-1175.

[17] S. Kinoshita, S. Isu, G. Kaneko, H. Yamada, T. Hara, Y. Itoh, S. Watabe, The occurrence of eukaryotic type III glutamine synthetase in the marine diatom Chaetoceros compressum, Mar. Genomics 2 (2009) 103-111.

[18] K. Backman, Y.M. Chen, B. Magasanik, Physical and genetic characterization of tthe glnA-glnG region of the Escherichia coli chromosome, Proc. Natl. Acad. Sci. USA 78 (1981) 3743-3747.

[19] R.W. Davis, D. Botstein, J.R. Roth, Advanced Bacterial Genetics: A Manual for Genetic Engineering, Cold Spring Harbour Laboratory, New York, 1980.

[20] M.M. Bradford, A rapid and sensitive method for the quantitation of microgram quantities of protein utilizing the principle of protein-dye binding, Anal. Biochem. 72 (1976) 248-254.

[21] R.A. Bender, K.A. Janssen, A.D. Resnick, M. Blumenberg, F. Foor, B. Magasanik, Biochemical parameters of glutamine synthetase from Klebsiella aerogenes, J. Bacteriol. 129 (1977) 1001-1009.

[22] U.K. Laemmli, Cleavage of structural proteins during the assembly of the head of bacteriophage T4, Nature 227 (1970) 680-685.

[23] H. Blum, H. Beier, H.J. Gross, Improved silver staining of plant proteins, RNA and DNA in polyacrylamide gels, Electrophoresis 8 (1987) 93-99.

[24] M.D. Abramoff, P.J. Magelhaes, S.J. Ram, Image processing with ImageJ, Biophotonics Int. 11 (2004) 36-42.

[25] A. D'Arcy, C. Elmore, M. Stihle, J.E. Johnston, A novel approach to crystallising proteins under oil, J. Cryst. Growth 168 (1996) 175-180.

[26] M. Ohi, Y. Li, Y. Cheng, T. Walz, Negative staining and image classification powerful tools in modern electron microscopy, Biol. Proced. Online 6 (2004) 23-34.

[27] A. Chirokov, Adobe ${ }^{\circledR}$ Photoshop ${ }^{\circledR}$ FFT/iFFT plugin, 2005

[28] F.C. Gibson III, A.B. Onderdonk, D.L. Kasper, A.O. Tzianabos, Cellular mechanism of intraabdominal abscess formation by Bacteroides fragilis, J. Immunol. 160 (1998) 5000-5006.

[29] R.E. Miller, E. Shelton, E.R. Stadtman, Zinc-induced paracrystalline aggregation of glutamine synthetase, Arch. Biochem. Biophys. 163 (1974) 155-174.

[30] Z.F. Burton, D. Eisenberg, A procedure for rapid isolation of both groE protein and glutamine synthetase from E. coli, Arch. Biochem. Biophys. 205 (1980) $478-488$.

[31] V.L. Tsuprun, E.J. Boekema, A.V. Pushkin, I.V. Tagunova, Electron microscopy and image analysis of the GroEL-like protein and its complexes with glutamine synthetase from pea leaves, Biochim. Biophys. Acta 1099 (1992) 67-73.

[32] C. Bartolucci, D. Lamba, S. Grazulis, E. Manakova, H. Heumann, Crysta structure of wild-type chaperonin GroEL, J. Mol. Biol. 354 (2005) 940-951. 